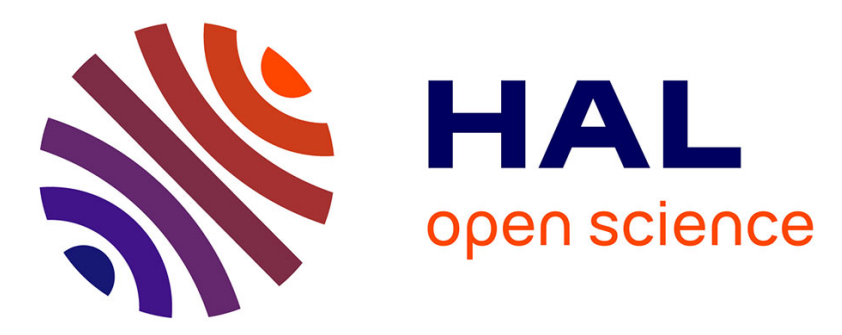

\title{
Risk perception and risk-taking among skateboarders
} Laurence Kern, Annie Geneau, Sophie Laforest, Alex Dumas, Benoit Tremblay, Claude Goulet, Sylvie Lepage, Tracie A. Ta Barnett

\section{To cite this version:}

Laurence Kern, Annie Geneau, Sophie Laforest, Alex Dumas, Benoit Tremblay, et al.. Risk perception and risk-taking among skateboarders. Safety Science, 2013, pp.370-375. 10.1016/j.ssci.2013.08.009 . hal-01177288

\section{HAL Id: hal-01177288 \\ https://hal.science/hal-01177288}

Submitted on 23 Jul 2015

HAL is a multi-disciplinary open access archive for the deposit and dissemination of scientific research documents, whether they are published or not. The documents may come from teaching and research institutions in France or abroad, or from public or private research centers.
L'archive ouverte pluridisciplinaire HAL, est destinée au dépôt et à la diffusion de documents scientifiques de niveau recherche, publiés ou non, émanant des établissements d'enseignement et de recherche français ou étrangers, des laboratoires publics ou privés. 


\section{Risk perception and risk-taking among skateboarders}

- Laurence Kerna,,

- Annie Geneaub,

- Sophie Laforest ${ }^{\mathrm{b}}$,

- Alex Dumas

- Benoit Tremblayd,

- Claude Goulete,

- Sylvie Lepagef,

- Tracie A. Barnett ${ }^{g, h}$

\section{Abstract}

Skateboarding is considered to be a high risk activity. Although many studies have identified risk factors associated with skateboarding injuries, few have provided detailed in-depth knowledge on participants' psychological dispositions towards risk behaviors. The aim of this study was to identify individual factors associated with risk perception and risk-taking among skateboarders. Telephone interviews were conducted with 158 skateboarders (mean age $=18.1$ years) recruited in 11 Montreal skateparks. Age, self-efficacy, previous injuries, fear of being injured, sensation seeking and experience level were all included in two linear regression models that were run for risk perception and risk-taking. Age, experience level, sensation seeking, and risk perception are significant explanatory variables of risk-taking. Results show that sensation seeking was the only significant factor associated with risk perception. These results allow for a better understanding of the behavior of skateboarders, they highlight the importance of impulsive sensation seeking in risk perception as well as risktaking. This study characterizes skateboarders who take risks and provides additional information on interventions for injury prevention.

\section{Keywords}

- Risk-taking;

- Risk perception;

- Sensation seeking;

- Self-efficacy;

- Skateboarding 


\section{Introduction}

In recent years, the popularity of risk-oriented sports has increased in our society despite the number of injuries and the risks associated with these activities (Llewellyn et al., 2008, Pain and Pain, 2005 and Turner et al., 2004). In this respect, skateboarding has often been singled out because of the incidence and type of injuries (fractures, contusions, strains, sprains, and lacerations of the lower arm, lower leg, head, and face) (Rainville et al., 2010). Even if the number of deaths is small, the number of injuries sustained is large. As within many areas of North America and Europe, skateboarding has become a mainstream activity in the province of Québec, especially amongst male youths (Hamel and Goulet, 2006). A government report on injuries indicated that the annual rate of reported injuries that require medical attention was 26/1000 skateboarders in Québec (ranked 12th among sports activities) and 35/1000 skateboarders in Montreal (ranked 7th) (Hamel and Goulet, 2006).

Although many studies have identified several risk factors associated with skateboarding injuries (i.e., speed, equipment failure, level of experience, risk perception, age, practice locations), few have provided detailed and in-depth knowledge on participants' psychological dispositions towards risk behaviors. Yet, knowledge of these psychological factors can enable us to better understand how risk takers function, which in turn allows us to propose more effective interventions. From the perspective of sports injury prevention, a study on risk perception, self-efficacy and sensation seeking among skateboarders would allow us to better understand risk-taking in this sport. Indeed, Llewellyn et al. (2008) demonstrate the relationship between self-efficacy and risk-taking in rock climbing and other risk sports, while Kontos (2004) shows that low levels of perceived risk are associated with a significant increase in risk of injury. We conducted a study among 158 skateboarders to explore the factors associated with risk perception and risk-taking, by integrating elements of two concepts, namely sensation seeking and self-efficacy. Zuckerman's (1974) concept of sensation seeking is often put forward to explore risk behaviors; more recently, Llewellyn et al. (2008)analyzed the relationships between Bandura's (1977) social cognitive theory variables and risk behaviors.

\subsection{Risk-taking and risk perception}

Risk-taking can be defined as a decision involving a choice that is characterized by a degree of uncertainty with respect to the probability of failure or success 
(Michel et al., 2001). More specifically, risk-taking behavior has been defined "as either a socially unacceptable volitional behavior with a potentially negative outcome in which precautions are not taken (e.g., speeding, drinking and driving) or a socially accepted behavior in which the danger is recognized (competitive sports, skydiving) (Turner et al., 2004, p. 93).

According to Davis-Berman and Berman (2002), understanding risk-taking cannot be done without reflection on the perceived risks. For these authors, "perceived risk involves a subjective perception of the potential for injury or death inherent in an activity" (Davis-Berman and Berman, 2002, p. 306). More specifically, several studies have reported an association between risk behaviors and risk perception in sports and recreation activities (Ajcardi and Therme, $\underline{2008}$ and Curry and Youngblade, 2006). Thus, risk perceptions are linked to risk behaviors on a theoretical level as much as on an empirical level (Fishbein, 2003 and Halpern-Felsher et al., 2004).

The classic theoretical prediction of the relationship between risk perception and risk-taking is the negative (protective) correlation between risk-taking and risk perception, which means that the greater the risk, the less likely the person will be to engage in the behavior in question (Mills et al., 2008). In contrast, the opposite positive relationship is also observed: a person with an increased perception of risk will have a greater tendency to engage in risky behavior (Johnson et al., 2002, Mills et al., 2008 and Reyna and Farley, 2006). For instance, throughout adolescence, risk-taking has been shown to be instrumental to social integration (Michel et al., 2001). In a qualitative study on skateboarding injuries, adult skateboarders were less inclined than their younger counterparts to take risks due to the threat that injuries pose to their ability to work and to their economic independence (Dumas and Laforest, 2009). In this respect, risk-taking is context-specific and dependent on perceived benefits.

\subsection{Self-efficacy, sensation seeking, risk perception and risk-taking}

Bandura (1997) puts forward the hypothesis that people take risks because they feel that they are capable of handling the situation and have a sense of increased self-efficacy. Self-efficacy refers to "the belief in one's capabilities to organize and execute the courses of action required to manage prospective situations" (Bandura, 1997, p. 3). People with high levels of perceived capabilities are more likely to challenge themselves, mobilize efforts and persist for a longer period of time in the face of difficulty (Bandura, 1997). ForSlanger and Rudestam 
(1997), executing a risky manoeuvre depends on the degree of confidence in one's ability to succeed. Hence, athletes demonstrating high self-efficacy would be more likely to engage in risky behaviors. From this perspective, the most important decisional factor among risky sports enthusiasts is self-efficacy. Several studies also analyzed the relationship between sensation seeking and risk-taking in sports activities (De Vries et al., 2009, Kajtna and Matej, 2004 and Slanger and Rudestam, 1997). For instance, sensation seeking has been found to be a significant correlate of a range of high-risk behaviors, such as drinking, smoking, drug use, and risky sexual behaviors (Zuckerman (1979)). For Zuckerman (1994, p. 27), sensation seeking is a trait that is defined as "the seeking of varied, novel, complex, and intense sensations and experiences and the willingness to take physical, social, legal, and financial risks for the sake of such experiences". In general, research tends to place sensation seeking in the category of impulsive and deviant conduct and associate it with other personality variables such as extraversion (Carton, 2005).

Several studies examined the roles of self-efficacy and sensation seeking in risktaking behaviors (Llewellyn and Sanchez, 2008, Llewellyn et al., 2008 and Slanger and Rudestam, 1997). Their conclusions suggest that the level of self-efficacy is higher among those who take extreme risks than those who take less risk. Slanger did not highlight a relationship between sensation seeking and risk-taking, while Llewellyn and his collaborators reported positive and significant associations between sensation seeking and risk-taking. These associations are attenuated, although still significant, when the authors take into account age, gender, and years of experience in the activity of choice (Llewellyn and Sanchez, 2008).

To our knowledge, there have not been any studies that explored the joint relationship of risk perception, risk-taking, self-efficacy and sensation seeking in skateboarding. It is the first time that these concepts have been studied together and we argue that this will enable us to better understand the perception of skateboarders By taking into account these variables, both dispositional (sensation seeking) and sociocognitive (risk perception, self-efficacy...), we can understand, explain and predict people's behavior. The sociocognitive and dispositional variables can serve as the impetus for proposing avenues for reflection in order to develop prevention programs. The objectives of this study were to identify the individual-level factors (age, socioeconomic status, experience, previous injuries, fear of being injured, self-efficacy and sensation 
seeking) associated with (1) risk perception and (2) risk-taking among skateboarders.

\section{Materials and methods}

\subsection{Research design and study population}

Objectives were addressed using a cross-sectional research design. Telephone interviews were conducted among 158 skateboarders aged 8-37 years.

Participants were recruited from 11 outdoor skateparks, comprising a representative sample of the 31 outdoor skateparks in Montreal. The selected parks were chosen in order to be as representative as possible of skateboarders and the skateboarding practice. They were located in different geographical areas of the city, in both supervised and unsupervised settings, characterized by different levels of difficulty, and frequented by groups of different socioeconomic status. Participants were recruited over two separate periods. First, in the summer of 2005, an initial study was conducted to build a profile of the injuries of all skateboarders who frequented skateparks. Research assistants recorded the address and telephone number of the 337 skateboarders in a registry, comprising the first pool of potential participants for the current study. A second recruitment period was conducted the following summer in order to improve the statistical power; 146 additional skateboarders were recruited in the 11 selected parks. In 2007, the project coordinator attempted to contact all 483 skateboarders identified in the summers of 2005 and 2006 to participate in the current study. In total, 272 individuals could not be reached (no answer/wrong number), 49 declined the offer to participate and four people did not meet the inclusion criteria (Speaking French or English, older than 8 years old, having skateboarded in the previous year, living in Montreal, having accepted to participate and returned the consent form). As such, 158 skateboarders completed the telephone questionnaire. All skateboarders who had reached the age of majority (18 years old), as well as the parents of the younger skateboarders, signed a consent form. This study was approved by the Ethics Committee of Université de Montréal.

\subsection{Measurement instruments}

The telephone questionnaire consisted of items that aimed to describe the characteristics and practice of skateboarders, as well as scales that were subject 
to psychometric validation (temporal stability $-n=38$-, internal consistency $n=158-$ ) and a back-translation (French and English). The temporal stability indicators were calculated for the first 38 participants who responded to the same questions in a two week interval, on average. Younger participants did not have difficulty responding to the questionnaires. Dependents and independents variables are introduced in the Table 1.

Table 1.

Dependents and independents variables.

Dependent variables

Risk perception

Risk-taking

Independent variables

Self efficacy

Previous injuries

Fear of being injured

Sensation seeking

Experience level.

Age and socioeconomic level

Other variables (protective equipment...)

\subsubsection{Dependent variables}

\subsubsection{Risk perception}

Adaptation of skateboarding to the Risk of Injury in Sports Scale or RISSc (Kontos, 2000): "Please indicate how likely you think it is that the following events will happen to you while skateboarding, on a scale ranging from 1 (very unlikely) to 4 (very likely)". The first event was "Be injured in a collision with a car'. The scale comprised 23 items that were selected based on the literature and in collaboration with experts (including both researchers and skateboarders). The test-retest correlation was very good $(r=0.84)$, as was the internal consistency (alpha $=0.78)$.

\subsubsection{Risk-taking}

Adaptation of skateboarding to the Risk-taking Behaviors Scale (Kontos, 2004): "Please indicate, on a scale ranging from 1 (never) to 4 (frequently), how often you engage in the following behaviors when skateboarding", such as 'skateboarding on boulevards' and 'crossing intersections at a red light without slowing down'. The scale was comprised of 17 items selected from the literature 
and with a group of experts that provided dangerous risk-taking situations for skateboarders. The test had good metric qualities (correlation test-retest of 0.80 and Cronbach's alpha of 0.70 ). Scores ranged from 17 to 68 with larger scores indicating more risk-taking.

\subsubsection{Independent variables}

\subsubsection{Self-efficacy}

The Self-Efficacy for Physical Abilities ( Ryckman et al., 1982) scale was adapted to the practice of skateboarding: "I am going to read a series of statements regarding your skateboarding skills. Please tell me the extent to which they describe you on a scale of 1 (agree strongly) to 4 (disagree strongly)", for example: 'You have good control of your movements in all situations' or 'You have limited motor abilities'. This questionnaire was comprised of 10 items. The two-week test-retest correlation was 0.45 and the internal consistency was 0.70 . Lower scores indicating less self-efficacy (some items were reverse coded for scoring purposes).

\subsubsection{Previous injuries}

This item was defined by the number of injuries that required a visit to a health professional (Hamel and Goulet, 2006): "Since you have started skateboarding, how many injuries required a consultation with a health care specialist or someone specialized in alternative medicine?" The test-retest correlation was 0.83 .

\subsubsection{Fear of being injured}

The Gymnastics Fear Inventory ( Cartoni et al., 2005) was also adapted to skateboarding: "The following questions address your concerns about skateboarding. For each question, please tell me what describes you best on a scale of 1 (never) to 4 (very much)". This scale was comprised of five questions, for example: "Do you consider yourself to be an apprehensive skateboarder?" The test-retest correlation was 0.69 and Cronbach's alpha coefficient was 0.68. Larger scores indicating more fear. 


\subsubsection{Sensation seeking}

Sensation seeking was measured using the Impulsive Sensation

Seeking (ImpSS) scale of ZKPQ, short version ( Aluja et al., 2006). Respondents were required to respond to the following question: "This is a series of statements that people might use to describe themselves. I am going to read them. Please tell me whether or not they describe you. If you agree with a statement or decide that it describes you, answer TRUE. If you disagree with a statement or feel that it is not descriptive of you, answer FALSE. The ImpSS was comprised of 10 items, such as 'I often do things on impulse', eight of which were related to sensation seeking and two to impulsiveness. The psychometric qualities were good (test-retest correlation of 0.73 and Cronbach's alpha of 0.56 ). Lower scores indicated less impulsiveness.

\subsubsection{Experience level}

The questions related to months of experience and perceived skateboarding level were adapted from the study on risk behaviors in alpine skiing (Goulet et al., 2000): "For how many weeks, months or years have you been practicing skateboarding?"; "In which category of skateboarder would you place yourself: beginner, beginner-intermediate, intermediate, advanced or expert?" (Test-retest correlation of 0.83 for months of experience).

\subsubsection{Age and socioeconomic level}

To assess perceived economic status, participants were asked: "Compared with people of your age, would you say that your family's economic situation is (1) better than theirs; (2) the same as theirs; (3) worse than theirs (Lavallée, 2004)?

\subsubsection{Other variables}

Other data collected included skateboard practices (use of protective equipment, frequency of skateboarding on average per week).

\subsection{Data analyses}

First, descriptive analyses were conducted in order to characterize the population. Second, bivariate correlation analyses were conducted in order to explore the relationships between the independent variables (sensation seeking, self-efficacy, previous injuries, fear of being injured, age, socioeconomic status, 
level of skateboarding experience) and risk perception and risk-taking. Third, linear regression was carried out to explore the relationships between the independent variables and each of the dependent variables (i.e., risk perception and risk-taking). Two sets of models were tested, one for identifying factors associated with risk perception and the second for risk-taking. A priori, all independent variables were included in these models. All analyses were performed using SPSS version 14.0. A significance threshold of .05 was used and all tests were bidirectional.

\section{Results}

\subsection{Descriptive analyses}

The sample was composed of skateboarders with an average age of 18.1 years $(\mathrm{SD}=5.2)$, males $(95 \%)$, francophone $(91 \%)$ and students $(76 \%)$. The majority considered their socioeconomic status comparable to that of their peers $(71 \%)$, while $7 \%$ reported it to be less favorable. The majority among them believed that their experience was at an intermediate level (51\%) or advanced level (29\%). They reported skateboarding at least three times a week for the past 68.5 months on average (SD $=50.8$ months). The places most frequented were outdoor parks, streets and urban areas. More than $80 \%$ said that they never wore protective equipment other than a helmet, which was occasionally worn by half of them. They reported having consulted a health professional for 2.4 injuries on average $(S D=4.14)$.

Lastly (Table 2), the average score for: fear of injury was 11.0 (SD = 2.9; max score $=20)$, self-efficacy was $30.8(S D=3.5$; max score $=40)$, impulsiveness was $6.4(\mathrm{SD}=2.1$; $\max$ score $=10)$, risk perception was $57.6(\mathrm{SD}=7.6$; $\max$ score $=92)$ and risk-taking was $40.9(\mathrm{SD}=8.1 ; \max$ score $=68)$ (see Table 2).

Table 2.

\begin{tabular}{ll|l|l|l|l}
\hline $\begin{array}{l}\text { Descriptive statistics PERC, COMP, HAB, PEUR and IMPSS } \\
\text { Variables }\end{array}$ & $M$ & S.D. & $N$ & Min-Max & Possible interval \\
\hline Risk perception & 57.6 & 7.55 & 154 & $35-76$ & 23 (low) -92 (high) \\
\hline Risk-taking & 40.94 & 8.13 & 158 & $23-57$ & 17 (low) -68 (high) \\
\hline Self-efficacy & 30.75 & 3.54 & 158 & $20-40$ & 10 (low) -40 (high) \\
\hline Fear & 10.96 & 2.85 & 157 & $05-19$ & 5 (low) -20 (high) \\
\hline Impulsive sensation seeking & 6.36 & 2.08 & 157 & $01-10$ & 1 (low) - 10 (high)
\end{tabular}

The samples size varies because of missing data for certain questions. 


\subsection{Bivariate analyses}

According to $\underline{\text { Table } 3}$, there is a significant and positive relationship between risk behavior and the following variables: risk perception, impulsive sensation seeking, level of experience and injuries. Risk perception is positively and significantly associated with sensation seeking. Self-efficacy is positively and significantly linked to experience, injuries and age, but negatively linked to fear. We observe that sensation seeking is positively correlated to experience and injuries.

Table 3.

Correlations matrix.

Risktaking behavior Self Sensation Experience

Risk .33 perception

Risk-taking

Self efficacy

Fear

Sensation seeking

Experience level Injuries efficacy Fear seeking level Injuries Age

\begin{tabular}{l|l|l|l|l|l|}
-.055 & .13 & .21 & .048 & .13 & .03
\end{tabular}

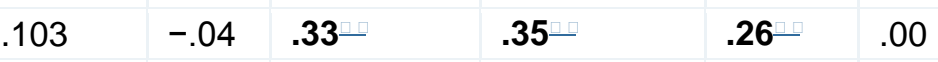

$-.18 \quad .09$

.36

$.27-\quad .23$

$p \leqslant .05$. $-.022$

.032 $.08 \quad .18 *$

.22 $.21-\quad .132$

$.37-\quad .40$ .37

$p \leqslant .001$.

\subsection{Regression analyses}

We tested two models, each of which included all individual level correlates. Sensation seeking ( $\beta=0.722, p \leqslant .05$ ) was the only significant variable associated with risk perception. The model tested explained $7.8 \%$ of the risk

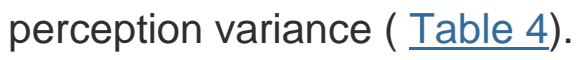

Table 4.

Linear regression analysis - risk perception $(N=150)$. Non-standardized coefficients

Perception

B SD

Significance level $P$ 


\section{Non-standardized coefficients}

\begin{tabular}{|c|c|c|c|}
\hline \multirow[b]{2}{*}{ Perception } & & \multirow{2}{*}{$\begin{array}{l}\text { Significance leve } \\
P\end{array}$} \\
\hline & $B$ & SD & \\
\hline Age & -.049 & .14 & .716 \\
\hline Experience level & -.059 & .92 & .949 \\
\hline Injuries & .211 & .16 & .201 \\
\hline Self-efficacy & -.205 & .20 & .308 \\
\hline Fear & .324 & .22 & .150 \\
\hline Sensation seeking & .722 & .31 & .020 \\
\hline Socio-economic status & -.603 & 1.23 & .626 \\
\hline
\end{tabular}

Risk-taking was regressed on all individual level correlates including risk perception. Several independent significant correlates were identified, including age $(B=-0.32, p \leqslant .05)$, level of experience $(B=3.52, p \leqslant .001)$, sensation seeking $(B=0.67, p \leqslant .05)$, and risk perception $(B=0.30, p \leqslant .001)$. The association between previous injuries and risk-taking was almost significant ( $\beta=0.29, p=.06)$. The model tested explained $31 \%$ of the variance in risk-taking ( Table 5).

Table 5.

Linear regression analyses - risk-taking $(N=150)$.

Non-standardized coefficients

\begin{tabular}{|llll} 
& & & \multicolumn{2}{l}{ Significance level } \\
\cline { 2 - 3 } Risk-taking behavior & $B$ & SD & $P$ \\
\hline Age & $-\mathbf{0 . 3 2}$ & $\mathbf{0 . 1 3}$ & $\mathbf{. 0 1}$ \\
\hline Experience level & $\mathbf{3 . 5 9}$ & $\mathbf{0 . 8 6}$ & $\mathbf{. 0 0 1}$ \\
\hline Injuries & 0.29 & 0.15 & .06 \\
\hline Self-efficacy & -0.05 & 0.19 & .78 \\
\hline Fear & -0.25 & 0.21 & .23 \\
\hline Sensation seeking & $\mathbf{0 . 6 7}$ & $\mathbf{0 . 2 9}$ & $\mathbf{. 0 2}$ \\
\hline Risk perception & $\mathbf{0 . 3 0}$ & $\mathbf{0 . 0 8}$ & $\mathbf{. 0 0 1}-$ \\
\hline Socio-economic status & 0.72 & 1.15 & .53 \\
\hline
\end{tabular}

$R^{2}=.31(F=7.76, p \leqslant .001)$.

$p \leqslant .05$.

$p \leqslant .001$. 


\section{Discussion}

The objectives of our study were to identify independent correlates of risk perception and of risk-taking. These objectives were addressed using a crosssectional design among 158 skateboarders recruited in 11 parks that were representative of the parks in the City of Montreal. Although the only significant explanatory factor of risk perception was the "impulsive sensation seeking" trait, several other factors were significantly associated with risk-taking (age, experience, injuries, sensation seeking, and risk perception).

We found that age was negatively associated with risk-taking. Data showed that older skateboarders take less risk than the younger skateboarders. As argued by Dumas and Laforest (2009), this result can be explained by the heightened awareness of the consequences of injury as skateboarders age; injuries are thus perceived as obstacles to their financial independence, making injury prevention a fundamental concern. With respect to level of experience, our results were similar to those of Kontos (2004), revealing that a higher level of experience was also significantly associated with more risky behaviors. Thus, skateboarders who have experience and who consider themselves to be at a high level have more confidence and therefore take more risks.

We also noted a boarderline positive relationship between the number of injuries with consultation and risk-taking $(p=0.06)$. As such, the more the skateboarders were injured during their practice, the more they tended to take risks. It may also mean that those who reported taking more risks were injured more often during these activities. It may also indicate that an activity is perceived to be risky if they were injured while undertaking that activity. The past injuries reported by participants may have been resolved successfully, thus having little impact on their risk-taking. Morrongiello (1997) indicates that children tend to avoid repeating only those behaviors that lead to more severe injury outcomes. The findings of this study suggest that self-efficacy is marginally associated with risk-taking ( $B=-0.21, p=.31$ ) and when perception of self-efficacy increases, risk perception decreases. The link is almost null with risk-taking. As such, in this context, there is little connection between the estimation of one's skills and the perception of risk or risk-taking. However, it is also possible that our adaptation of a scale developed for gymnastics was not valid for skateboarding. These results on the relation between risk-taking and self-efficacy are not entirely in line with Llewellyn and Sanchez (2008) or with Bandura's hypotheses (1997). 
Our results highlighted a positive relationship between risk perception and risktaking, which coincides with the works of Reyna and Farley (2006) and Johnson et al. (2002). Evidence suggests that a higher level of perceived risk by skateboarders is related to greater risk-taking (Mills et al., 2008). Risk is stimulating, valued, and perceived as a means of integrating oneself and of being validated by one's peers (Turner et al., 2004). If these anticipated benefits exceed the perceived risks of injury, the risky behavior is more likely to be adopted (Davis-Berman and Berman, 2002). Moreover, one study showed that benefit perception predicted risky behaviors to a greater extent than risk perception (Rolison and Scherman 2003). Thus, an evaluation of the perceived benefits likely allow for a better understanding of risk-taking among the skateboarders in our study.

Positive relationships were highlighted between sensation seeking, risk perception and risk-taking. Thus, skateboarders who ranked higher on the sensation seeking score were more likely to perceive risks to be present and to take greater risks. As such, perception of risk is related to sensation seeking. However, Zuckerman et al. (1978) highlighted a negative relationship between the total score for sensation seeking (Sensation Seeking Scale Form V) and perception of risks (Zuckerman, 1979). This is in line with the reports of other authors: "some adolescent sports participants maintain high or inflated levels of estimation of ability in spite of previous injuries or that they are injured more often, due to their confidence. The past injuries reported by participants may have been minor or may have been resolved successfully, thus increasing estimation of ability" (Kontos, 2004, p. 453). A few reasons may explain this divergent result. First, impulsive sensation seeking was used in the current study in contrast to the global scale of sensation seeking used in other studies. Thus, direct comparisons between the results of our study and those of other studies may not be possible. Second, when evaluated in a general manner, risk perception is also called risk identification (Millstein and Halpern-Felsher, 2002) and participants were asked to identify the risks that they perceived in a hypothetical situation ("If you are skateboarding in the street, what are the chances that you will be in a collision with a car?"). Millstein et al. (2002) define this as conditional risk. Another evaluation method consists of asking participants to evaluate the risk of being injured while skateboarding, as we did in our study ("What are your chances of being in a collision with a car?"), which Millstein et al. (2003) refer to as unconditional risk. Depending on the way risk perception is 
measured, the relationship between risk perception and risk-taking differs. Though not exhaustive, these appear to be the most relevant explanations for these divergent findings.

\section{Strengths and limitations}

This study sought to better understand risk-taking behaviors among skateboarders. Original aspects of our study included our investigation of (1) the relationship between the predictive variable, risk perception, and the explanatory variables, self-efficacy and impulsive sensation seeking, as well as (2) the relationship between the predictive variable, risk-taking, and the explanatory variables, risk perception, self-efficacy and impulsive sensation seeking. In addition, we used psychometrically validated tools that were adapted to the specific behavior being studied.

There are certain limitations that need to be mentioned. First, it is a crosssectional analysis, precluding statements about causality. In addition, although questionnaires were validated, measures were self-reported, which may have led to a social desirability bias. With respect to injuries, participants had to report the number of injuries in their lifetime, and recall errors may be possible. Regarding self-efficacy, the adaptation carried out may not have been valid for this study. In addition, risk perception is a complex construct that is challenging to measure, and it would merit particular attention with respect to its operationalization for subsequent studies. Lastly, although precautions were taken to ensure a representative sample, the study's participants may not be a perfect statistically representative sample of the skateboarders from the 31 Montreal parks. The skateboarders from this study are not representative of the entire population of skateboarders as they come from outdoor municipal skateparks only.

\section{Conclusion}

This study was conducted within a perspective of sports injury prevention among skateboarders in order to better understand their risk-taking as it pertains to this activity. It enabled us to characterize skateboarders who take risks and to identify the variables that are apparently the most significant in explaining the variance of this behavior. A typical skateboarder who will take risks while skateboarding is younger with an intermediate level of experience. He has a tendency to value impulsive sensation seeking and positively perceives the risks that he will be taking. 
This typical profile of risk-taking skateboarders takes into account the role of personality traits (impulsive sensation seeking) and socio-cognitive variables (self-efficacy, with which fears of injury, previous injuries, age and experience level are associated). These results allow for a better understanding of the behavior of skateboarders and highlight the importance of impulsive sensation seeking in risk perception and risk-taking, while taking a series of variables into consideration.

These results also allow us to understand that people who are already injured during their skateboarding practice are people who remain at risk. In fact, even if they perceive greater risks, they take more risks and are more at risk for injuring themselves. As such, prevention programs should target these people, and sports instructors must pay particular attention to them.

\subsection{Perspectives}

Although there have been many studies on the determinants of sport injuries, three reasons support the need for additional data on specific groups who are at risk for skateboarding injuries. First, epidemiological data on sports injuries show that skateboarding remains popular among youth and that it is linked to high levels of injury when compared to other sports (Hamel and Goulet, 2006). Second, there is a number of new sociocultural and environmental factors that may increase the incidence and severity of injury and that should be considered. Research in sports policy indicates a growing trend in 'lifestyle sports', that is, new forms of physical activities that are associated with youth and characterized by their delocalization from traditional sporting venues, risk-taking and an emphasis on hedonism, self-expression and creativity (Tomlinson et al., 2005 and Wheaton, 2004). The potential impact of this trend should be considered since motor vehicle accidents with street skateboarders have been identified as major causes of serious injuries (Forsman and Eriksson, 2001, Lustenberger et al., 2010 and Osberg et al., 1998). The results of this study could also be applied to other physical activities in order to understand risk behavior. Third, knowledge of specific groups who are at risk for serious sports injuries is also needed in order to better adapt injury prevention strategies. Results of this study suggest that there will be significant challenges in promoting low-thrill activities and helmet use in young male skateboarders. They show that injury prevention experts will have to be creative in their efforts to reduce risk- 
taking of a target population (teenage boys) who are specifically seeking thrills through risk-taking.

There were no actual or potential conflicts of interest, including financial and personal conflicts, or any other relationships with people or organizations within 3 years of starting the submitted work that could inappropriately influence, or be perceived to influence, this work. 